\title{
Exploitation Minière, Facteur De Recompositions Socio-Economiques Dans La Sous-Préfecture De Hiré (Côte d'Ivoire)
}

\author{
Kouadio Kouassi Nicolas \\ Université Alassane Ouattara de Bouaké (Côte d’Ivoire) \\ Département de Sociologie-Anthropologie
}

doi: 10.19044/esj.2016.v12n17p287 URL:http://dx.doi.org/10.19044/esj.2016.v12n17p287

\begin{abstract}
Expected as a development opportunity, the construction of the mine of Bonikro (sub-prefecture of Hiré), has led to significant changes in the life of the local communities. In an initial context marked by a dynamic economy focused on agriculture, the implantation of the mining industry has not only generated new opportunities, but also economic constraints in the face of which local communities have developed adaptation strategies which have led locally to socio-economic reconstruction. The present study attempts to reveal these new behaviors and to analyze the logic which underlies them.
\end{abstract}

Keywords: Gold, artisanal mining, industrial mine, sustainable development, socio-economic reconstruction

\section{Résumé}

Attendue comme opportunité de développement, la construction de la mine de Bonikro (sous-préfecture de Hiré) a engendré des changements notables dans la vie des communautés locales. Dans un contexte initial, marqué par une dynamique économique portée par la production agricole, l'implantation de l'industrie minière a généré de nouvelles opportunités mais aussi des contraintes économiques face auxquelles les communautés locales développent des stratégies d'adaptation qui ont conduit localement à la recomposition socioéconomique. La présente étude tente de révéler ces comportements nouveaux et d'analyser les logiques qui les sous-tendent.

Mots clé : Or, orpaillage, mine industrielle, développement durable, recomposition socioéconomique 


\section{Introduction}

Cette contribution porte sur les recompositions socioéconomiques induites localement par la construction de la mine de Bonikro (cote d'Ivoire). La mine de Bonikro (sous-préfecture de Hiré), est le résultat de deux années de recherches démarrées en 1996 et qui ont établi la présence d'or économiquement exploitable en 1998. En janvier 2007, un permis d'exploitation (PE 032) a été accordé à la compagnie sud-africaine EQUIGOLD-CI, pour l'exploitation de ce gisement. La construction de l'usine a commencé en mai 2007 et la production effective de l'or en octobre 2008. Cette mine est pionnière pour la Côte d'Ivoire au regard de la modernité des technologies utilisées. Pour accroitre sa production, Newcrest Mining Limited, une compagnie australienne qui a racheté la mine de Bonikro, a poursuivi l'exploration dans la zone, et découvert des gisements satellites. Cette compagnie a obtenu en 2013, un nouveau permis pour exploiter ces gisements. Il s'agit du permis PE 044 dans la sous-préfecture de Hiré. Cette activité minière s'inscrit dans un contexte économique local traditionnellement marqué par l'agriculture.

Sur le terrain, le constat est que l'implantation de la mine a occasionné des opportunités, mais aussi des contraintes économiques pour les communautés locales. Face à ces opportunités et contraintes, les communautés locales déploient des stratégies individuelles et collectives à partir des ressources dont elles disposent pour tirer profit du nouvel environnement économique. Ces stratégies sont à la base de la recomposition socioéconomique amorcée localement et fondent l'intérêt de cette étude. La spécificité de cette étude est de mettre l'accent sur l'approche locale (communautés riveraines) de l'impact du secteur minier, contrairement aux approches plus globalisantes vues sous l'angle macroéconomique et géopolitique. L'objectif est de montrer comment l'installation de la mine de Bonikro a généré des opportunités et des contraintes économiques face auxquelles les communautés locales développent des stratégies d'adaptation qui entrainent la recomposition socioéconomique en cours dans la souspréfecture de Hiré.

\section{Méthodologie de recherche}

Cette étude a été réalisée à partir de deux types de données. Il s'agit de données provenant des enquêtes de terrains et de la recherche documentaire. Selon les objectifs de cette étude, nous avons eu recours à deux outils de collecte à savoir: les entretiens semi-directifs et les questionnaires. Le choix pour l'entretien semi- directif répond au souci de collecter des informations susceptibles de décrire et de comprendre la perception des communautés elles-mêmes et le sens qu'elles donnent à la dynamique socioéconomique amorcée localement. Il s’agit de faire ressortir 
leurs différentes logiques d'actions à partir des argumentations qu'elles donnent. Au total huit (8) focus groups ont été réalisés avec des groupes de jeunesses, de femmes et de paysans. Chaque focus group a vu la participation d'au moins huit (8) personnes. L'aspect quantitatif de l'étude a nécessité deux (2) questionnaires. L'une a été distribuée à 300 orpailleurs et l'autre à 55 paysans déplacés et indemnisés. En filigrane, l’objectif du deuxième questionnaire était d'appréhender les transformations socioéconomiques à travers la capacité des paysans à reconstituer leur capital de production. A cet effet, les rubriques suivantes ont été renseignées : identification du paysan, l'étendue de la superficie détruite, les stratégies de reconstitution du capital de production économique.

Toutefois, des entretiens complémentaires ont été nécessaires pour compléter les données issues des entretiens ciblés. Il s'agit des entretiens avec le service local du ministère de l'agriculture, de la mairie et de la souspréfecture. Dans cette lucarne, des entretiens informels jugés utiles à l'enrichissement des données ont eu lieu. Il s'agit d'entretiens avec des artisans, des commerçants etc. pour avoir leur opinion sur les changements survenus du fait de la présence de la mine.

\section{Champ géographique de cette étude}

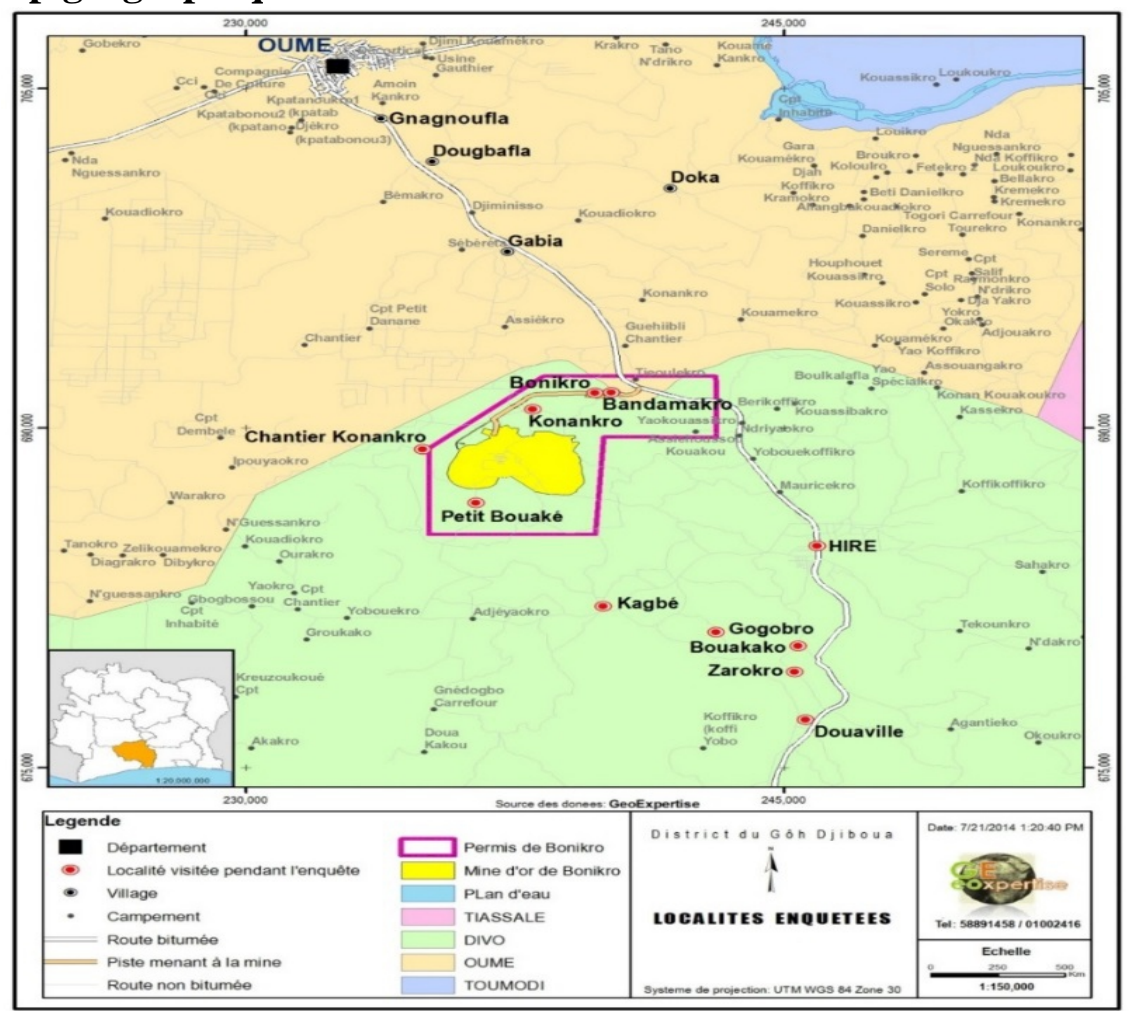

Source : Newcrest, 2014 


\section{Ancrage théorique de l'analyse}

L'installation d'une usine minière à Bonikro (sous-préfecture de Hiré) a créé un nouvel environnement social et économique dans la région. Ce nouvel environnement a inévitablement une influence dans les habitudes de la population locale. Dans ces conditions, l'installation de la mine se présente comme un facteur de changement social. Cette étude s’est appuyée par conséquent sur la théorie du changement social et du développement selon laquelle, l'analyse des actions de développement et des réactions populaires ne peut être disjointe de l'étude des dynamismes locales, des processus endogènes, ou des processus informels de changement (Olivier de Sardan, 1995). Il s'agit d'analyser les changements observables dans les comportements des populations depuis l'installation de la mine. Le changement social est défini par Rocher (1977) comme « toutes transformations observables et vérifiables dans le temps qui affectent d'une manière qui n'est pas provisoire la structure ou le fonctionnement d'une collectivité et qui en modifie le cours de son histoire». La prégnance des comportements opportunistes, l'orpaillage, les nouveaux rapports sociaux guidés par les enjeux générés par la mine, sont les variables sur lesquelles nous nous sommes basés dans cette approche théorique pour analyser le changement socioéconomique en vigueur à Hiré.

\section{Resultats}

\section{Description du milieu d'étude avant l'introduction récente de l'activité minière.}

Données générales d'occupation des terres dans la sous-préfecture de Hiré

Le peuplement de la sous-préfecture de Hiré s’est fait en trois phases essentielles. La première phase a été animée par l'arrivée des Dida, considéré comme communauté autochtones. Ils auraient fait l'exode du Ghana avec les Akan, c’est pourquoi on les appelle les " mamini » (Zézé, 1981; Bernus, 1962). La deuxième phase migratoire a été conduite par un groupe de Baoulé arrivé dans la sous-préfecture pour l'activité d’orpaillage. Toutefois, cette exploitation traditionnelle de l'or de grande envergure va connaître une fin vers les années 1940, du fait de l'arrivée des mineurs français. L’arrivée des mineurs français à Hiré autour des années 40, a entrainé une troisième vague migratoire composée d'ouvriers maliens et burkinabé notamment. A la fermeture des mines (artisanale et semi-industrielle), la plupart de ces ouvriers mineurs migrants sont restés pour se reconvertir en producteurs de café-cacao, devenue l'activité économique phare de la région. La recherche de terres fertiles pour la création de plantations de cacao et de café a été déterminante dans le flux migratoire qu'a connu le petit village d'HiréWatta, à l'instar de toutes les zones pionnières de la culture du cacao (Ruf, 
1995). En effet, l'essor de la culture de cacao que Ruf a appelé « booms du cacao », a contribué fortement à la dynamique démographique des zones forestières de la Côte d'Ivoire. Ces différents modes de migration ont permis au petit village de Hiré-watta de s'agrandir et de connaître une dynamique démographique remarquable, à l'origine de son érection en circonscription administrative (sous-préfecture) en 1977, puis en commune rurale en 1985.

Depuis 2007, avec la construction de la mine d'or de Bonikro, la commune de Hiré fait face à une nouvelle vague de flux migratoire. Il s’agit d'une part, d'employés de la mine, venus d'autres régions du pays et aussi de pays étrangers notamment le Ghana; et d'autre part, d'orpailleurs venus d'autres régions du pays et surtout des pays voisins (du Burkina Faso notamment).

\section{Les secteurs d'activités économiques}

L'activité économique dominante à Hiré est l'agriculture. Elle est à la base du peuplement de toute la sous-préfecture. A côté de cette activité économique prédominante, il existe d'autres activités dont le dynamisme dépend toujours de l'agriculture. Il s'agit de l'artisanat, du commerce et d'autres activités non agricoles. La chasse qui était l'activité principale des Bida, est aujourd'hui l'activité phare de certains Dioula. Organisés en groupe, ils pratiquent la chasse au filet avec des chiens de chasse. Les petits métiers (artisanat) sont aussi pratiqués à Hiré. Il s’agit de la couture, la coiffure, la mécanique auto, vélo, moto etc. Dans l'unique marché de la ville se pratique le commerce de vivriers, de vêtements et autres produits cosmétiques.

\section{Exploitation minière, entre contraintes et opportunités.......... Occupation des terres par la mine industrielle}

L'exploitation minière à ciel ouvert est une activité qui consomme de grands espaces (Thune, 2011). L'emprise foncière de la mine à ciel ouvert est une réalité à la mine de Bonikro. L'espace utilisé pour l'exploitation de la mine de Bonikro couvre une superficie de 847,07 hectares (administration et clôture de sécurité y comprises). Cette infrastructure économique occupe des surfaces qui abritaient initialement des activités économiques des populations riveraines. A travers l'agriculture, ces populations tirent leur subsistance. Ce sont environ 150 paysans initialement propriétaires de ces terres qui ont été partiellement ou totalement expropriés et indemnisés. La photo $n^{\circ} 1$ ci-après, donne un aperçu de la fosse de Bonikro et la destruction du couvert végétal qu'elle occasionne. L'emprise des opérations de la mine de Hiré quant à elle, s'étend sur 640 hectares pour plus de 1000 exploitants expropriés. Cette occupation du sol par les activités minières crée des contraintes économiques énormes pour ces paysans. 


\section{Aperçu de la fosse de Bonikro}

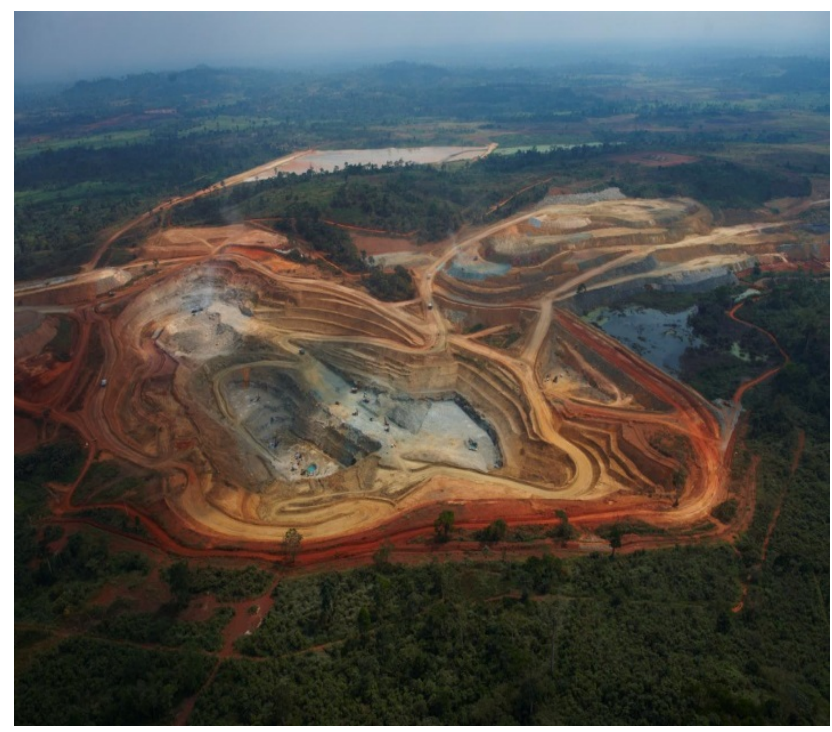

Source: Newcrest Mining,Avril 2012

\section{Des paysans sans terre, la vie au quotidien}

Pour garantir leur sécurité alimentaire, les paysans cultivent chaque année du vivrier qui est destiné à l'autoconsommation. Si la production du cacao permet d'avoir de l'argent pour les autres charges du ménage, la production de cultures vivrières permet quant à elle, de subvenir aux besoins alimentaires de la famille. Les jachères disponibles permettent d'assurer cette sécurité alimentaire. Avec l'expropriation des terres, les paysans sont désormais obligés d'acheter la nourriture quotidienne. Selon nos enquêtes, $44,4 \%$ des paysans expropriés de leurs terres en partie ou entièrement, avouent acheter de la nourriture pour satisfaire leurs besoins alimentaires. Leur stratégie de sécurité alimentaire se trouve ainsi limitée par la perte des terres. Dans les campements déplacés de Bandamakro et Bonikro, l’achat de nourriture se perçoit comme une manifestation palpable de la transformation de leur mode de vie habituel. Eux qui produisaient pour se nourrir et nourrissaient même la population de la commune, sont aujourd'hui contraints à acheter ce qu'ils doivent manger. Même le bois de chauffage, qui sert à cuire le repas est acheté. Aussi, du fait de l'idée que les populations de ces campements sont pour la plupart bénéficiaires d'indemnisation et donc “riches”, le prix des denrées alimentaires est particulièrement élevé dans ces campements. Cette situation justifie l'opinion locale selon laquelle les populations de Bonikro seraient rendues vulnérables par l'exploitation minière, et est à la base des revendications des populations déplacées de Bonikro et Bandamakro. Selon ces populations elles méritent d'être assistées 
en termes de fourniture de vivres par la compagnie minière. D’ailleurs, ils fondent leur droit à l'assistance alimentaire sur les promesses (non écrites) de certains responsables de la mine lors de l'élaboration du projet de délocalisation. Selon les populations de Bonikro, Monsieur X de la compagnie minière a promis de leur « fournir de la nourriture tout le temps ». Mais depuis l'ouverture de la mine, aucune action dans ce sens n’a été menée.

Une autre contrainte est l'accès au crédit. Les paysans ont l'habitude d'accéder à un système de crédit non conventionnel entre eux ou auprès d’autres opérateurs économiques, notamment les acheteurs de café-cacao. Ce système de crédit comprend: le gage (garantie), «l'assoumbo» ou intérêt à 100\%, l’intérêt en kilogrammes de cacao, l'obligation de vente à un acheteur. - « la garantie » (gage) est un mode de crédit qui permet d'avoir du crédit en contre partie de la cession d'une partie ou de la totalité de la plantation pour une durée déterminée d'accord partie. La durée minimum est de 1(un) an et dépend de la qualité et de l'étendue de la plantation en comparaison du montant perçu.

- «L'assoumbo » est un crédit à 100\% d’intérêt. Le montant prêté est remboursé pendant la traite à $100 \%$ d'intérêt.

- « Le prêt à intérêt en kilogrammes de cacao » est une voie d’accès au crédit moyennant remboursement en une quantité de cacao à la traite (de l'espèce en nature). La correspondance est de 20.000f CFA pour 100 kilogrammes de cacao.

- «L'obligation de vente à un acheteur » est une forme de contrat de crédit qui lie le producteur et l'acheteur de cacao. Il consiste à prendre un crédit auprès d'un acheteur en contrepartie de la fidélisation de vente de toute la production et ce, malgré le prix pratiqué par ce dernier.

La condition principale d'accès à ces formes de crédit est la possession d'une plantation qui sert d'aval. Ne plus disposer de plantation signifie, perdre la possibilité d'accès au crédit. Les paysans expropriés de leurs plantations sont donc limités dans les stratégies de crédit qui leurs permettaient de supporter les périodes de crise ou de solutionner les problèmes brusques (maladie, décès, frais scolaires, etc.) qui nécessitent de débourser des sommes importantes.

\section{Expropriation foncière : une perte ou un gain dans la perception des communautés locales}

Les paysans reconnaissent leur faiblesse face à l'Etat, en ce qui concerne l'expropriation foncière pour utilité publique. De ce fait, ils n’opposent généralement pas de résistance face à l'occupation de leurs terres par la compagnie minière. Les premières vagues de compensations agraires obtenues par les paysans de Bonikro ont d'ailleurs, suscité de l'admiration 
des bénéficiaires au sein de la communauté. Chacun veut que son champ soit touché par les activités de la mine pour bénéficier des compensations. Le simple passage de l'exploration ou l'information (pas souvent fondée) de l'arrivée probable de la mine dans une zone, faisait naître de l'espoir chez les paysans de la zone visée. Ils trouvent là, une opportunité de devenir riche. Cette perception est partagée par plusieurs paysans. Ils suivent de ce fait de près, les mouvements d'exploration de la mine. Pendant la durée de notre présence à la mine au département SDE, nous avons pu voir des paysans formuler des doléances pour que la mine occupe toute leur terre lorsqu'une partie seulement est touchée par les opérations de la mine. Certains même nous demandaient si la mine arriverait dans leur zone. Lors de la délimitation de certains terrains visés par les activités de la mine, on pouvait lire la joie dans le discours des paysans concernés « enfin le grand bas-fond » disaientils. Certains formulaient déjà des projets sur l'utilisation future de l'argent qui sera perçu suite à l'indemnisation. Même si quelques paysans manifestent le refus de l'occupation de leur terre, ce n'est pas pour la crainte de la perte de leurs plantations ou de leurs terres, mais c'est parce qu'ils estiment que le barème de l'indemnisation n'est pas satisfaisant. Dans l'ensemble, les gros montants obtenus par les bénéficiaires de l'indemnisation constituent un gain pour les paysans qui n'ont jamais reçu de leur vie à partir d'une production annuelle, de telles sommes.

\section{Des stratégies locales d'adaptation.}

Alors que les terres non exploitées (terrain nu) n’ont pas une grande valeur monétaire dans l'indemnisation (250.000 FCFA/ha), les paysans ont trouvé un palliatif à travers la culture par opportunité appelé localement « piège ». La culture par opportunité consiste à mettre en valeur les terres en jachère dans l’optique de bénéficier de l'indemnisation lorsque le terrain sera visé par les activités minières. Ce comportement est devenu pour les paysans, la principale stratégie pour tirer profit de la mine. L'objectif principal n'est pas de cultiver pour une production économique, mais plutôt, d'attendre patiemment que la mine passe par là, cause des destructions et indemnise les plantes détruites.

Toutes les jachères ne sont pas systématiquement mises en valeur. La logique du « piège » obéit à un cadre stratégique qui consiste à mettre en valeur les terres qui sont visées par les activités (exploration et exploitation) de la mine. Pour cela, plusieurs indicateurs sont à la disposition des paysans. Ce sont: les marques de peinture sur les arbres et les rubans rouge-blanc qui annoncent le passage future des machines de l'exploration ou de l'exploitation. L'information sur la probable annexion d'une zone par la compagnie provient aussi des employés à la mine. Une fois cette information disponible, toutes les jachères sont immédiatement mises en valeur. 
A Hiré commune, l'annonce d'une probable exploitation des gisements (prospect), matérialisée par l'Étude d’Impact Environnemental et Social (EIES) début 2010, a entrainé remarquablement le développement de ces comportements opportunistes. Chaque individu, pourvu qu'il ait accès à la terre, a posé son piège. L'emprise du projet minier est actuellement “piégés”. Sur une distance de plus de 3km à l’Est et au Sud-est de Hiré, où ont été découverts les gisements, s'étendent des plantations de tecks et d'anacardes, créées de façon opportuniste pour bénéficier d'un éventuel dédommagement.

Les pièges ne concernent pas seulement les cultures. Les maisons servent aussi «d'appât» pour la communauté. Les zones d'habitation visées par le projet d'extension de la mine à Hiré sont systématiquement mises en valeur. En 2010, année de confirmation de l'effectivité du projet minier de Hiré à travers les consultations publique dans le cadre de l'étude d'impact, La ville de Hiré qui a connu un lotissement anarchique remarquable. Des propriétaires situés sur l'emprise du projet ont morcelé leur terre en lots urbain dans des zones hors du plan d'urbanisation de la ville. Cette initiative leur permettrait de maximiser les gains pendant l'indemnisation car ils espéraient gagner doublement. D'abord avec l'indemnisation des cultures situées sur ces lots puis avec les lots qu'ils projetaient négocier entre 2500 et $5000 \mathrm{FCFA} / \mathrm{M}^{2}$ contrairement au terrain rural qui coûte $200 \mathrm{~F} \mathrm{CFA} / \mathrm{M}^{2}$. Finalement, après plusieurs discussions entre l'opérateur minier, les propriétaires de ces “terrains urbains" et l'administration locale, le prix du $\mathrm{M}^{2}$ de terrain urbain est arrêté à 500 FCFA, ce qui est toujours supérieur à celui du terrain rural.

Des spéculations spéciales, étrangères dans le système agricole local sont mises en culture par les paysans. Ce sont le teck, l'anacarde et le jatropha. Le choix de ces plantes répond à plusieurs facteurs de justification: (i) le coût à l'indemnisation, (ii) la résistance aux intempéries (sécheresse, feux de brousses) et (iii) des atouts agronomiques que présentent ces plantes. Selon le barème d'indemnisation adopté par la compagnie, ces plantes ont un avantage économique comparativement aux autres plantes, notamment le cacao et le café. En plus de cet avantage économique, ces plantes, (excepté le jatropha qui résiste peu au feu), résistent mieux à la sécheresse et aux feux de brousses qui sont fréquents dans la région. En outre, ces plantes sont moins exigeantes en termes de qualité de la terre. Elles réussissent aisément sur les terres arides. Dans l'espace périurbain de Hiré marqué par une forte dégradation du sol due à un usage excessif, ces plantes sont les mieux indiquées pour garantir un profit. 


\section{Exploitation artisanale de l'or, une opportunité économique révélée par la construction de la mine de Bonkro}

L'artisanat minier occupe une part très importante dans l'économie informelle en Afrique. Pratiqué dans au moins 25 pays sur le continent, l'artisanat minier offre plus de 2,5 millions d'emplois directs et fait vivre plus de 20 millions de personnes (Communities and Small-Scale Mining, 2004). Cette activité offre de meilleures possibilités pour la création d'emplois par rapport aux grandes entreprises minières (Nations Unies, 2009). L'artisanat minier se présente ainsi comme un véritable moyen de lutte contre la pauvreté en Afrique. Certains pays africains ont pris conscience de l'importance de ce secteur dans l'économie nationale. Ils prennent dès lors des dispositions légales et institutionnelles pour l'inclure dans le processus de développement. Au Cameroun (Nguepjouo et Manyacka, 2008) au Burkina-Faso (Gueye, 2001) au Ghana (Appiah, 1998) par exemple, il existe des structures en charge de l'organisation et de la formation des orpailleurs, de la production, de l'achat etc. Ces structures sont en général des moyens de l'Etat pour assurer un suivi régulier de l'artisanat minier au niveau de la production, de la commercialisation et de la maîtrise des effets indésirables de ce secteur d'activité.

A Hiré, l'orpaillage a refait surface après environ un siècle de fermeture des premiers sites, simultanément avec la construction de la mine industrielle de Bonikro en 2007. Débuté avec un seul site et une dizaine d'acteurs, l'orpaillage est aujourd'hui de loin l'activité économique phare de la commune de par le nombre d'acteurs mobilisés (environ 3000 personnes) la multiplication des sites dans la sous-préfecture. Les orpailleurs sont d'origines socioprofessionnelles très variées. L'opportunité économique que présente l'orpaillage, et la morosité de leur activité initiale les poussent à pratiquer l'orpaillage soit temporairement, soit de façon indéterminée. On trouve donc dans le rang des orpailleurs, des paysans, des commerçants, des hommes de métiers etc. Cependant, les commerçants et les paysans sont les plus nombreux avec respectivement $22,5 \%$ et $20,7 \%$. Ils sont suivis par les artisans ou "hommes de métiers"' avec $15,1 \%$ et les élèves $10,9 \%$. Les orpailleurs professionnels sont peu nombreux avec $9,1 \%$ de notre échantillon. 


\section{Répartition des orpailleurs selon l’origine socioprofessionnelle.}

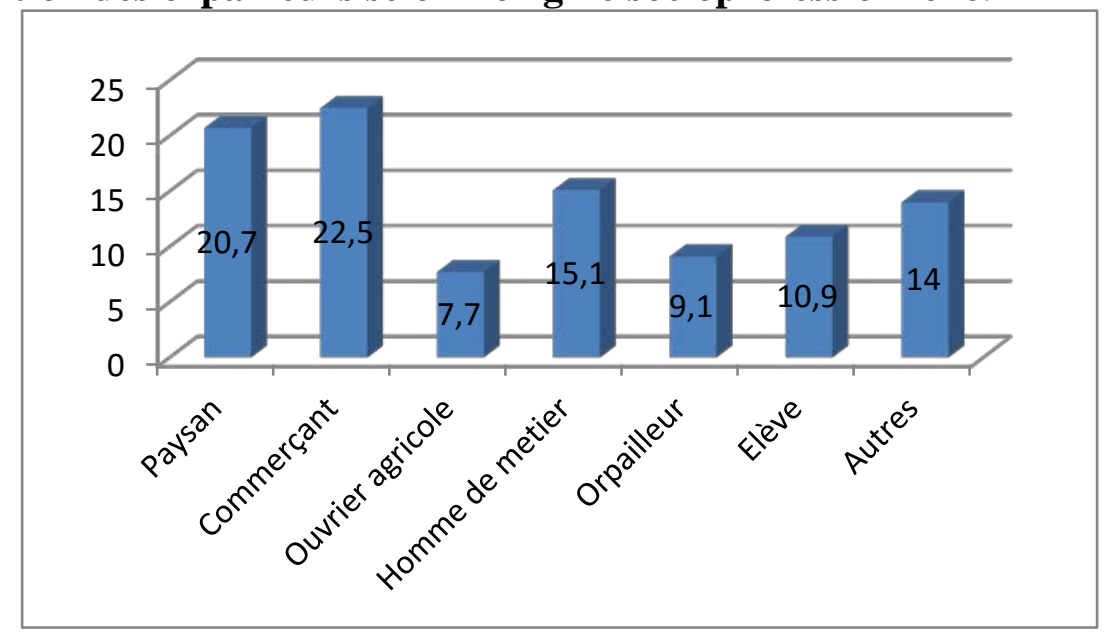

Source: Kouadio, 2012.

Toutefois, la plupart des orpailleurs ne comptent pas s'investir durablement dans l'orpaillage. Pour ces derniers, l'artisanat minier est une réponse circonstancielle au manque d'emploi et au besoin de subsistance dont ils font face actuellement. Cette catégorie d'orpailleurs fréquente les sites d'orpaillage de façon occasionnelle. Ils s'orientent vers l'orpaillage pour avoir un revenu “rapide'” pour répondre à un besoin spontané ou encore obtenir un capital financier important pour le réinvestir dans l'activité principale. Sur les sites, ils opèrent de façon individuelle ou encore offrent leur force de travail à des orpailleurs professionnels (plus ou moins constitué) comme ouvriers journaliers. Dans cette catégorie, on compte des personnes qui disent être dans cette activité faute de mieux. "Tu vois, il n'y a rien d'autre à faire. On se débrouille ici, actuellement, c'est l'orpaillage qui nous permet de nourrir nos femmes et nos enfants " (D.M : orpailleur; Août 2012). Ces orpailleurs occasionnels sont pour la plus part ressortissants de Hiré. Ils saisissent l'opportunité économique que présente l'artisanat minier lorsque leur activité initiale tourne au ralentir. Les élèves trouvent aussi dans l'orpaillage une opportunité pour subvenir à leurs besoins. Certains élèves issus de familles modestes, fréquentent les sites d'orpaillage pendant les vacances ou à la rentrée, les samedis, dimanches et jours fériés pour faire de l'orpaillage à titre individuel ou comme ouvriers journaliers dans une unité de production plus grande. Le revenu obtenu leur permet d'aider leurs parents à assurer leur scolarité et leur subsistance. Cette catégorie d'orpailleurs occasionnels pratique l'orpaillage en attendant de trouver une activité économique plus lucrative et plus sécurisée. 


\section{Des stratégies d’adaptation.... à la recomposition socioéconomique Fragilisation de l'économie agricole}

Le recrutement par la mine de la main d'œuvre locale pour l'exécution temporaire de travaux de manœuvres (casual) est un facteur de la raréfaction de la main d'œuvre agricole. Ce recrutement est une promesse de la direction générale de la compagnie minière. Il s’agit en réalité d’une stratégie qui vise à atténuer la pression de la demande d’emploi à la mine. « [....] pour les travaux de manœuvre, nous n’irons pas chercher des gens ailleurs ; nous embaucherons les jeunes de Bonikro. ». Ce recrutement ne se limite pas au seul village de Bonikro. Il s’étend à toute la sous-préfecture de Hiré. Le salaire journalier de cet emploi temporaire à la mine est de 3.750 FCFA. Ce qui est largement supérieur au salaire que perçoit un manœuvre agricole la journée fixé à 1.500-2.000 F CFA. Cet avantage comparatif du revenu journalier à la mine explique l'attrait de la main d'œuvre locale pour la mine au détriment de la main d'œuvre agricole. Les emplois temporaires à la mine entrainent aussi la perte de la main d'œuvre familiale. En effet, les jeunes qui habituellement travaillaient comme aide des parents dans les exploitations familiales, sont recrutés comme employé “casual” à la mine. Cette situation indigne les paysans qui constatent sans pouvoir rien n'y faire, la fuite de la main d'œuvre agricole. Tel fut le cas d'un paysan interviewé dans le campement de Bonikro: «Les jeunes ne travaillent plus pour nous. Même nos propres enfants préfèrent travailler à la mine que d'aller au champ » (K.K.M : paysans à Bonikro, octobre 2012). La raréfaction de la main-d'œuvre agricole salariée et non salariée est un facteur clé à la base de la transformation du système de production local. Le manque de la maind'œuvre agricole contraint les paysans à diminuer sensiblement leurs parcelles d'exploitation ou à débourser plus d'argent pour engager la maind'œuvre. En effet, du fait des emplois temporaires à la mine, les coûts des prestations de la main-d'œuvre agricole ont augmenté.

La pratique de l'orpaillage reste également un facteur déterminant dans la raréfaction de la main d'œuvre agricole à Hiré. Cela se justifie par son avantage économique par rapport à l'agriculture en termes de revenu. En tant qu'ouvrier orpailleur, le salaire journalier (2.000-3.000 FCFA) est de loin plus avantageux que le salaire d'ouvrier agricole (1.000-1.500 FCFA). En plus, la pratique de l'orpaillage a permis à certains anciens ouvriers agricoles de s'installer à leur propre compte en tant qu'orpailleur. Cette nouvelle opportunité leur garantit une liberté par rapport au contrat d'ouvrier agricole qui les obligeait à travailler rigoureusement selon un calendrier qui leur laisse peu de marge de manœuvre. Ainsi chaque jour, des milliers de « bras valides » prennent d'assaut les sites d'orpaillage pour soit, monnayer leur force de travail pour le compte des chefs de chantier ou des propriétaires de puits, ou encore, chercher de l'or pour leur propre compte. 
Toutes les autorités administratives rencontrées sont unanimes que l'orpaillage est la principale cause du désintéressement des paysans vis-à-vis de l'agriculture. Selon un responsable du service locale de l'administration de l'agriculture, l'orpaillage cause le plus grand « mal » à l'agriculture surtout périurbaine à Hiré.

"Ils font les trous partout. Les espaces sur lesquels on pratiquait l'agriculture périurbaine, chou, oignon, tomate et autres sont occupés par les orpailleurs ». (E.M. du service local du ministère de l'agriculture, Avril 2013)

L'agriculture périurbaine en effet, avait une place très importante dans la structure économique locale. Il s'agit en réalité d'espaces cultivables, situés dans les environs (1à $3 \mathrm{Km})$ et des bas-fonds à l'intérieur de la commune qui permettaient à certaines personnes de pratiquer le maraîchage. Cette activité était la principale opportunité pour la communauté d'avoir rapidement un revenu. Les spéculations cultivées dans cette forme d'agriculture en générale, sont récoltables au bout de trois mois. Ces espaces sont aujourd'hui occupés par les sites d'orpaillage, limitant ainsi les possibilités de pratiquer cette activité économique. Le tort causé à l'agriculture ne se limite pas à l'occupation de l'espace par les sites d'orpaillage. Il s'étend aussi à l'occupation des acteurs (paysans).

\section{Activité minière, facteur de redéfinition de la richesse}

Initialement, dans les communautés paysannes de la Sous- préfecture de Hiré, le "riche" est une personne qui avait une grande production cacaoyère. Le critère de richesse se déterminait à la pesé de la production. Celui qui fait régulièrement un bon tonnage de cacao ou de café est considéré comme riche. Cette grande production dépendait de la superficie de l'exploitation, de la qualité de l'exploitation et de la main d'œuvre dont dispose le chef de l'exploitation. Certains paysans se sont ainsi démarqués des autres en termes de revenu annuel faisant d'eux les plus “riches".

L’indemnisation a chamboulé cette stratification sociale. Désormais, ceux qui sont vus comme " riches " sont les personnes bénéficiaires d'indemnisation. D'ailleurs, on les appelle localement les « boubous » ou millionnaires. Certains d'entre eux ont obtenu des montants avoisinant la centaine de million. Ces montants n'ont jamais été obtenus auparavant pendant une campagne de cacao dans l'histoire des paysans. Cette situation crée localement une nouvelle stratification sociale. Le riche n'est plus perçu par le seul indicateur de production annuelle de cacao ou de café, mais surtout celui qui bénéficie d’une indemnisation. 


\section{Employé à la mine, nouveau modèle de réussite sociale}

Avant l'implantation de la mine de Bonikro, les personnes perçues comme modèle de réussite sociale, c'est-à-dire ayant un revenu mensuel, permanent et « consistant » étaient les fonctionnaires et agents de l'Etat (enseignants, gendarmes et policiers et quelques commerçants prospères). Désormais, avec la mine, seuls les employés de la mine sont les mieux vus en termes de réussite sociale. On les appelle abusivement les travailleurs de la «SODEMI » ou encore « les miniers ». Ils sont identifiables à partir de leur uniforme orange à bandes réfléchissantes. Les témoignages avérés ou non sur leurs salaires mensuels sont les raisons de l'opinion que se font les gens de Hiré sur la situation financière des travailleurs de la mine. A cela s'ajoute, le mode de vie des mineurs qui vient confirmer cette opinion. Les travailleurs de la mine, en effet, possèdent pour la plupart une moto ou une voiture. Ils habitent les quelques "belles" maisons de la ville et constituent les fidèles et meilleurs clients des maquis-bars de la ville. Ce mode de vie fait des mineurs, le modèle de réussite sociale auquel aspire la plupart des gens de la Sous-préfecture.

\section{Discussion}

Le débat autour du rôle de l'exploitation minière dans le processus de développement durable des pays du Sud est factuel. En effet, l'exploitation minière est perçue par certains optimistes, comme le secteur d'avenir qui sortira l'Afrique de l'ornière de la pauvreté et du sous-développement. Cette perception est nourrie aussi bien sur le plan international (par les institutions de Breton Wood) comme à l'intérieur même des pays qui se targuent de posséder un sous-sol riche en ressources minières. Lors d'une conférence à Cape Town en Afrique du Sud en septembre 2005, un Directeur exécutif de la Banque Mondiale, Paulo Gomes affirmait ceci:

"The mining sector is set to play a key role in the World Bank's goal to alleviate poverty in Africa by 2015...If we use mining as the key driver for growth, then we would become less dependent on $\$ 50$ billon (in aid) that I am not sure will even come » (Tredway, 2005: p 6)

«Le secteur minier est appelé à jouer un rôle clé dans l’objectif de la Banque Mondiale de réduire la pauvreté en Afrique d’ici 2015... Si nous utilisons l'exploitation minière comme le principal moteur de croissance, alors nous serons moins dépendant des 50 milliard de dollars (d'aide) dont je ne suis pas sûr qu'ils soient disponibles ». (Notre traduction)

Cet optimisme placé dans le secteur extractif comme "délivreur" de l'Afrique de l'étau de la pauvreté est aussi partagé par les dirigeants africains. C'est pourquoi, dans les Documents Stratégiques de Réduction de la Pauvreté (DSRP), une part belle est faite au secteur minier comme raison pour espérer à un lendemain meilleur pour l'Afrique (Campbell, 2008). Dans 
son discours lors de l'inauguration de la mine d'or de Tongon, le président ivoirien disait ceci:

"L'inauguration de la mine d'or de Tongon est l'un des premiers jalons que nous posons pour réaliser notre ambition de faire du secteur des mines, un pôle majeur de développement économique et social de notre pays. En effet notre pays dispose de ressources minières importantes : du fer, du manganèse, de l'or ou encore du nickel latérite, deuxième réserve mondiale [...]. Leur mise en valeur procurerait ainsi à l'Etat des redevances, des impôts et des taxes; elle représenterait de très gros investissements et générerait des dizaines de milliers d'emplois. [...]. Je me félicite donc du projet de la mine d'or de Tongon, qui, de par la taille de son gisement et l'importance des sommes investies, générera des revenus pour l'Etat et contribuera au développement de cette zone, qui en a tant besoin. ${ }^{28}$

La croissance des devises de l'Etat et l'amélioration des conditions de vie de la population en général et des communautés hôtes des projets miniers en particulier sont donc les objectifs visés par les politiques minières en Afrique.

Sur le terrain, cet optimisme semble être relativisé par le contraste observé dans les pays africains qui ont déjà quelques années d’expériences dans le secteur minier. Cela transparait dans les rapports de certaines ONG et observateurs dans ce secteur (FIDH, 2007; Campbell, 2008; Dupin et Mialaret, 2004; Egan, 2001; Fischer, 2006). Faisant un constat général sur les vingt dernières années du rôle joué par le secteur minier dans le développent en Afrique, Campbell Bonnie indique que : "Les retombées en termes de revenu pour les pays concernés et de création d'emplois ont été de manière générale décevantes. Les possibilités que le secteur minier joue un rôle de catalyseur menant à une transformation et un développement économique et social ne sont pas réalisées à travers les approches et reformes passées » (Campbell, 2008: p3). L’exemple de quelques pays africains, connus pour avoir un potentiel minier important conforte cette assertion de Campbell. En dépit de la progression constante de la demande de matières premières minières, les pays les plus riches en ressources minières sont parmi les plus pauvres dans le monde (Pain pour le Prochain, 2010). Le classement des pays par Indice de Développement Humain (IDH), effectué par le PNUD en 2014 place aux derniers rangs. Sur 187 pays, le Mali (troisième producteur d'or en Afrique), est classé 176ème; la République Démocratique du Congo (dont la richesse du sous-sol est abondante et diversifiée : premier producteur mondial de cobalt, premier producteur de diamant industrielle), classée 186ème; le Niger,(troisième

\footnotetext{
${ }^{28}$ Propos du président de la République de Côte d'ivoire lors de l'inauguration de la mine d'or de Tongon
} 
producteur mondial d'uranium), ferme la marche du classement, 187éme. Ces données donnent à croire que l'exploitation des ressources minières ne participe pas réellement au développement des pays dotés de ces richesses. Cette absence de corrélation entre richesse du sous-sol et le développement de certains pays est qualifiée par Sachs et Warner (2001) de "The curse of naturel resources » (traduit en français par : la malédiction des ressources naturelles).

\section{Conclusion}

Dans l'histoire du secteur minier, le dynamisme économique et social local est très souvent tributaire de l'ouverture et de la fermeture de la mine. Cette dépendance socioéconomique des régions minières à la vie de la mine trouve son explication dans les stratégies de développement initiées soit par la compagnie minière, l'Etat ou les communautés locales elles-mêmes. Cette politique, ne vise pas une intégration économique. Elle est généralement opportuniste et est liée à la durée de vie de la mine. L'économie minière sans liaison mécanique avec les autres secteurs d'activité ne peut engendrer une économie pérenne comme l'indique un rapport des Nations Unies (Nations Unies, 2009).

Attendue comme occasion de voir leur niveau de vie s'améliorer, du fait des diverses opportunités qu'elle offrirait, la présence de la mine n’a pas tardé à occasionner des changements au sein de la communauté. D'abord chez les paysans, de nombreuses inquiétudes liées à l'occupation des terres voient le jour. La construction de cette infrastructure a nécessité en effet, l'accaparement de la terre (environ 1500 hectares), principal moyen de production économique locale. Dans ce nouveau contexte, les paysans deviennent comme des "victimes " d'un développement minier. Sans terre, ils voient leur stratégie de subsistance s'effondrer. Face à cela et dans un dispositif de réglementation minière qui valorise peu les terres nues (jachère) dans l'indemnisation des dommages causés, les paysans ont développé des stratégies pour combler la perte de leur terre. Il s'agit principalement de la mise en valeur de terres nues avec des plantes nouvelles telles que le teck, le jatropha et l'anacardier avec pour seul objectif l'indemnisation. Ces stratégies ont profondément transformé la configuration et la dynamique agraire locale.

Par ailleurs, parallèlement à la mine industrielle, une frange de la population s'est lancée dans une exploitation artisanale illégale de l'or. Cette nouvelle activité a progressivement occasionné une immigration massive de populations étrangères à Hiré et favorisé l'émergence d'un secteur économique informel florissant. Hiré est passé d'une ville agricole à une ville minière, offrant plusieurs opportunités économiques. 


\section{References:}

ADDI, A. (Sans date): Khourigba: la problématique de développement d'une ville minière marocaine. [En ligne] http://www.khourigba.net/IMG/pdf/khourigba.pdf. Consulté le 12/08/2013. AKABZAA, T.M; SEYIRE, J.S. et AFRIYIE, K. (2007): Une façade brillante: les effets de l'exploitation minière sur la ville d'Obuasi et ses communautés environnantes. Third World Network-Africa, 119 p.

APPIAH, H. (1998): Organization of small scale mining activities in Ghana, in The journal of South African institute of mining and metallurgy, [En ligne] http://www.saimm.co.za/Journal/v098n07p307.pdf; Consulté le 23/05/2012.

BERNUS, E. et VIANES, S. (1962): Tradition sur l'origine des dida mamini du canton wata (subdivision de Divo, Côte d'Ivoire), In, note africaine trimestriel, n91, janvier 1962, pp. 20-23.

CAMPBELL, B. (2008): L'exploitation comme moteur du développement en Afrique: enjeux de responsabilité et d'imputabilité. [En ligne], http://www.web.net/emi/concertationgrandslacs/2008/2008_01_23_BCampbel l.pdf. Consulté le 02/10/2012.

CHAUVEAU, J-P (1997): Des "stratégies des agriculteurs africains" au "raisonnement stratégique" : histoire, usages et remise en question d'un concept pluri-disciplinaire. In, Thème et variation, pp. 179-217.

CHAUVEAU, J.P (1978): Contribution à la géographie historique de l'or en pays baulé (Côte d'Ivoire). In, journal des Africanistes, vol. 48, pp. 15-70.

ECKERT, C. (1991): Une ville autrefois minière : la Grande-Combe, étude d'anthropologie sociale, Thèse de doctorat, Université Paris V 'René Descartes', Sciences Humaines- Sorbone, 782 p.

EGAN, L. (2001): Les effets des grandes exploitations minières sur les collectivités chiliennes, [enligne] http://idlc.idrc.ca/dspace/handel/10625/6247. Consulté le 23/11/2012.

FIDH (2007): L'exploitation minière de l'or et les droits de l'homme au Mali [en ligne], www.fidh.org/IMG/pdf/MI477f.pdf. Consulté le 12/11/2013.

GASTON, J. (1913): Exploitation indigène de l'or en Côte d'Ivoire. In, bulletins et mémoires de paris, $\mathrm{XI}^{\text {ème }}$ série, tome 4 , fascicule $3-4$, pp. 372 375.

KABUYA, M. A. (2009): Impact de l'exploitation de diamant et de l'or dans la reconstruction socioéconomique de la province orientale (République Démocratique du Congo), CENADEP, 16 p.

KEITA, A. et al (2008): Communautés locales et "manne aurifère »: les oubliées de la législation minière malienne. Ed, Lorenzo cotula, 53 p.

LEBAS, L. (2010): Impacts de l'exploitation minière sur les populations locales et l'environnement dans le Haut-Ogooué, [en ligne], http://www.brain- 
forest.og/img/Impacts_exploitation_minière_HautOgooue.pdf. Consulté le 23/07/2012.

LEVIN, E. (2009): Climate change and ASM: the facts and implications. ACC 2009/ Mozambique/ASM: an opportunity for rural development. [En ligne] http://www.estellelevin.com/index.php/pubications. Consulté le 06-042012.

MOREL, Y. (2009): Afrique de l'Ouest: promesses ambigües des richesses minières. In Débats-courrier d'Afrique de l'Ouest, nº8-septembre-octobre 2009 pp. 9-14.

Nations Unies (2009): Rapport d'examen africain sur l'exploitation minière, Commission économique pour l'Afrique. Addisabeba (Ethiopie), sixième session du comité de la sécurité alimentaire et du développement durable (CFSSD-6), [En

ligne] http://www.uneca.org/csd/csd6/AfricanFeviewReport-onMiningSummuryFR.PDF. Consulté le 12/08/2012.

NIANGORAN-BOUAH, G. (1978): Idéologie de l'or chez les akans de Côte d'Ivoire et du Ghana. In, journal des Africanistes, vol.48, pp.127-140.

OLIVIER De SARDAN, J.P. (2001): Les trois approches en anthropologie du développement, In revue du Tiers monde, tom XLII, n¹68 d’Octobredécembre pp.729-754.

ORRU, J.F. et PELON, R. (2005): Développement durable : quelle place pour l'artisanat minier? In, géoscience, $\mathrm{n}^{\circ} 1$, janvier 2005. [En ligne], http://www.brgm.fr/dcenewsFiles?ID=117. Consulté le 19/09/2011.

RUF, F. (1995): Booms et crises du cacao: les vertiges de l'or brun. Ed. Karthala, 459 p.

THUNE, M. (2011): L'industrialisation de l'exploitation de l'or à Kalsaka, Burkina Faso : une chance pour une population rurale pauvre? EchoGéo, $n^{\circ} 17 / 2011$, [en ligne] http://echogeo.revues.org/12535. Consulté le 17/01/ 2013.

ZEZE, B.P (1981): Origine et mise en place des populations dida. In, Anales de l’Université d’Abidjan, série I : Histoire, n99, pp. 123-137. 